\title{
3D TOPOLOGICAL SUPPORT IN SPATIAL DATABASES: AN OVERVIEW
}

\author{
Syahiirah Salleh ${ }^{1, *}$, Uznir Ujang ${ }^{1,}$ and Suhaibah Azri ${ }^{1}$ \\ 1 3D GIS Research Lab, Faculty of Built Environment \& Surveying, Universiti Teknologi Malaysia, Johor, Malaysia \\ syahiirahsalleh78@gmail.com, \{mduznir, suhaibah\}@utm.my
}

KEY WORDS: 3D Topology, Spatial Database, Topological Rules, Topological Data Structure, Topological Model, 3D GIS.

\begin{abstract}
:
The storage of spatial data that consists of spatial and non-spatial properties requires a database management system that possesses spatial functions that can cater to the spatial characteristics of data. These characteristics include the geometrical shape, topological and positional information. Parallel to how geometries describe the shape of an object, topological information is also an important spatial property which describes how the geometries in a space are related to each other. This information describes the connectivity, containment and adjacencies of spatial objects which are the foundation for more complex analysis such as navigation, data reconstruction, spatial queries and others. However, the topological support provided by spatial databases varies. This paper provided an overview on the current implementations of topological support in spatial databases such as ArcGIS, QGIS, PostgreSQL and others. The native topology in most spatial databases was found to be 2D topology maintained by 2D topology rules with limited representation of 3D topological relationships. Consequently, 3D objects represented by 2D topology had to be decomposed into objects of lower dimensions. Approaches to implement additional topological support for spatial databases included the use of topological data models, data structures, operators, and rules. 3D applications such as 3D cadastre required more detailed representations of topological information which required a more comprehensive 3D topological data model. Nonetheless, comprehensive preservation of topological information also mandates voluminous storage and higher computational efficiency. Thus, the appropriate 3D topological support should be provided in spatial databases to accurately represent $3 \mathrm{D}$ objects and meet $3 \mathrm{D}$ analysis requirements.
\end{abstract}

\section{INTRODUCTION}

Modelling spatial objects in three-dimensional (3D) environment has now become common. With the existence of 3D data acquisition technology that can be used at the end-user level (i.e., LiDAR), 3D data has become accessible, and it simplifies the process of modeling 3D objects. Currently, the use of 3D spatial objects is used in various applications such as environmental modeling (Ledoux, 2008; Ujang et al., 2018), GIS-based Building Information Modeling (Atazadeh et al., 2017; Döllner and Hagedorn, 2007; Wan Abdul Basir et al., 2017; al., 2018) and 3D city modeling (Biljecki et al., 2017; Jovanović et al., 2020; Salleh et al., 2021). Even so, there are still various issues as the 3D integration of objects between various fields sometimes require further assessments.

An object which has a location that ties it to the space is referred to as spatial data. Real-world processes can be understood by acquiring information from the analysis of spatial data. Analysis often requires large volumes of spatial data. Therefore, spatial databases are specifically used to store, manage and edit data with spatial properties (Winstanley and Mooney, 2020). However, spatial data also has non-spatial attributes that describes other properties of an object. A spatial database which consists of a standard database management system and a spatial subsystem is capable to handle spatial and non-spatial attributes as well as perform spatial functions (Mella et al., 2019). A wellfunctioning spatial database is required to handle data storage, data management, spatial data model support, spatial queries support and spatial indexing (Yue and Tan, 2018).

A spatial subsystem is responsible for spatial data management and spatial functions such as spatial data model support, query language for spatial queries and spatial indexing. The manner in which spatial data is constructed and functions is determined by a schema defined by a spatial data model (Howari and Ghrefat,
2021). A query language is another function of the spatial subsystem that allows the creation of instances, data editing and spatial queries. The Structured Query Language (SQL) is a wellknown query language used to access the spatial database and execute queries. In order to access spatial objects, the spatial data are indexed to ensure efficient query processes. The spatial indexing function determines the specific order and arrangement of data which can be in terms of location, geometry, attributes or relationships of the spatial data.

As spatial objects have a location or position, the object is considered to reside in a topological space. Topology is defined as the study of topological transformations and the properties that remain unchanged by changes to the space (Worboys and Duckham, 2004). In other words, topological properties of objects such as adjacencies, connectivity and containment remain unchanged (Ellul and Haklay, 2006; McDonnell and Kemp, 1995). Similar to how geometric properties describe the shape of an object, topological properties are also crucial to describe how objects within a space are related. In terms of topology for spatial objects, topology can be described as intrinsic topology and extrinsic topology. Intrinsic topology refers to topological properties and connectivity within a single object while extrinsic topology refers to topological relationships between objects (Knoth, Atazadeh and Rajabifard, 2020). The uses of topology may differ between the two whereby intrinsic topology usually handles geometric or topological validation of an object while extrinsic topology addresses how objects are related.

This paper attempts to provide an overview on the current topological support available for spatial databases and discusses the implementation of 3D topology for spatial databases. This paper is divided into four subsequent sections. Section 2 addresses current maintenance of topology in spatial databases. Section 3 will present implementations of custom topological extensions for spatial databases. Next, Section 4 will put forth a 
discussion on 3D topological support in spatial databases. Finally, this paper will be concluded in Section 5.

\section{CURRENT MAINTENANCE OF TOPOLOGY IN SPATIAL DATABASES}

A GIS or Geographic Information System is often the simple and go-to solution for storing spatial data. A desktop GIS can be defined as a stand-alone GIS software that consists of storage, analysis, display, retrieval and update of spatial data (Maguire, 2008). A well-established desktop GIS is ESRI's ArcGIS which implements topology rules to define topological relationships between spatial objects. The 32 ArcGIS geodatabase topology rules defines valid topological interactions between spatial objects from $0 \mathrm{D}$ vertices up to $2 \mathrm{D}$ polygons. For instance, polygons must not have gaps between them, lines must not selfintersect, points must be disjoint and others. Figure 1 depicts the 32 topology rules implemented in ArcGIS.

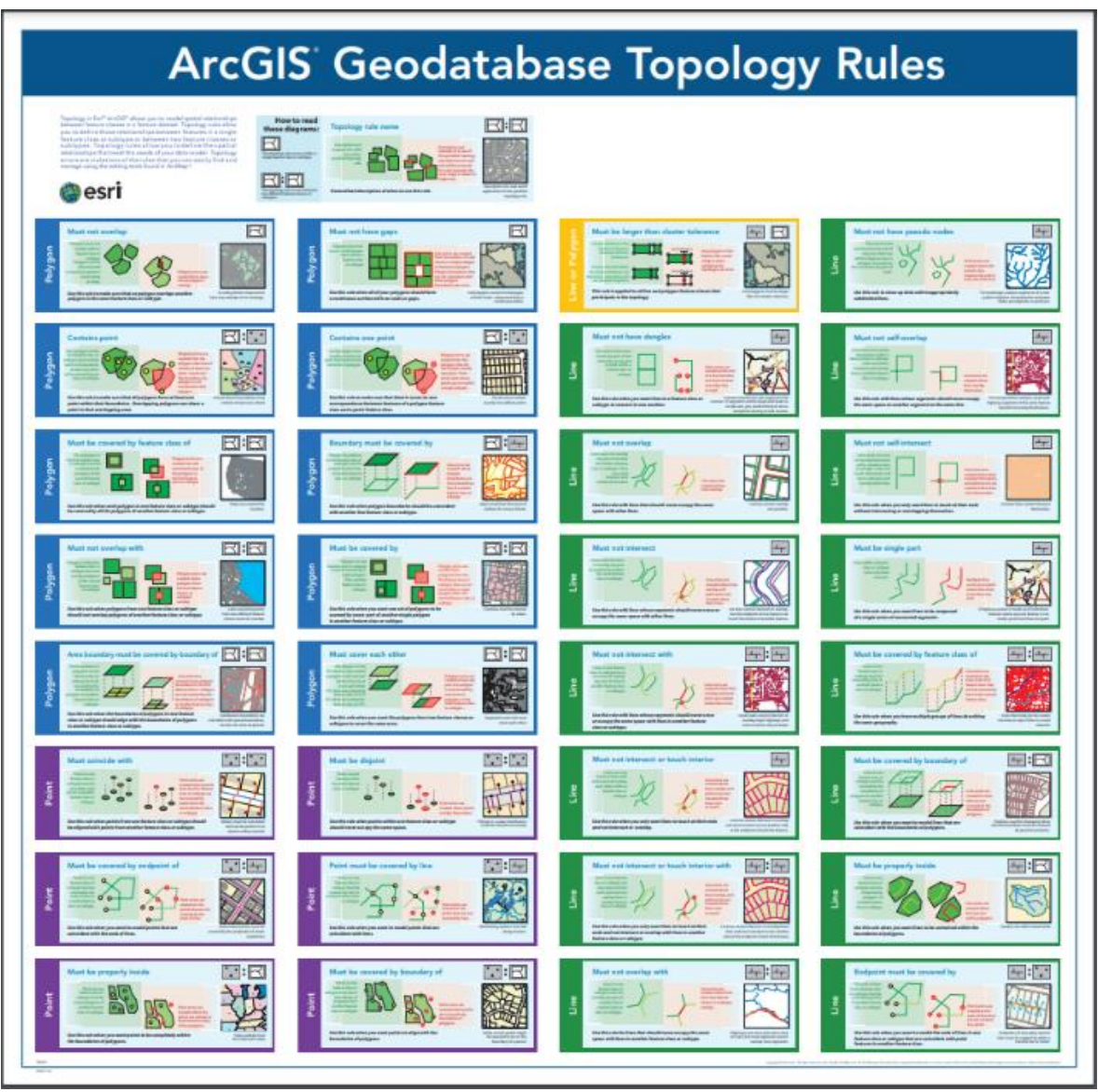

Figure 1. ArcGIS geodatabase topology rules (ESRI, 2010).

Another GIS software which is Quantum GIS (QGIS) also implemented topology rules based on topological rules to determine valid topological interactions for spatial objects of each dimension (QGIS, 2021). Spatial objects of each dimension has a pre-defined set of topological rules. The DE-9IM is also implemented in QGIS to determine topological relationships by examining the intersections between interiors, boundaries, and exteriors of spatial objects and the dimensionality of the intersection. The intersections are represented by -1 for empty or no intersection, 0 for intersection at $0 \mathrm{D}$ point, 1 for intersection at $1 \mathrm{D}$ line and 2 for a $2 \mathrm{D}$ polygon intersection. Figure 2 illustrates an example of "Overlaps" topological relationship between 2D regions based on the DE-9IM.

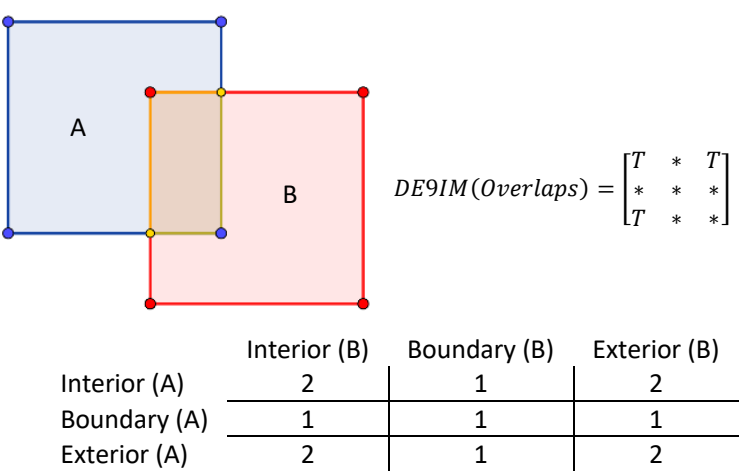

Figure 2. Example of "Overlaps" between 2D regions based on DE-9IM.

Similarly, SuperMap utilises topology rules as definitions of how spatial objects interact. SuperMap implemented different sets of topology rules where 6 rules were defined for $0 \mathrm{D}$ points, 14 rules for $1 \mathrm{D}$ lines and 10 rules for 2D regions (SuperMap, 2017). An 
additional 5 topology rules also define topological interactions between spatial objects of different dimensions.

A DBMS or database management system can also be used to store, manage, edit and update spatial data. In spatial databases, a DBMS is often assisted by spatial extensions to facilitate spatial data and spatial functions. PostgreSQL is an object-relational DBMS that can cater to spatial data with the inclusion of the PostGIS extension. The main language used to access the spatial database is SQL but PL/pgSQL, and C language can also be supported. A few topological rules were implemented in the extension which facilitated validation of geometries. The topological rules returned errors which describes invalid spatial objects. Similarly, Oracle DBMS can also support spatial data with the extension of object-relational Oracle Spatial. However, topology is handled by Oracle Spatial as a separate layer which explicitly represents topological primitives such as node, edge and face. The topology can be built based on topology data or spatial geometries. In building the topology layer, the topological primitives must adhere to specifications of valid geometries. Table 1 presents a summary for the topological component of spatial databases.

\begin{tabular}{|c|c|c|c|c|c|c|}
\hline \multirow{2}{*}{\multicolumn{2}{|c|}{ Spatial Database }} & \multirow{3}{*}{$\begin{array}{c}\begin{array}{c}\text { Topology } \\
\text { Model }\end{array} \\
\text { ArcGIS } \\
\text { Geodatabase } \\
\text { Topology } \\
\text { Rules }\end{array}$} & \multicolumn{4}{|c|}{ Number of Rules } \\
\hline & & & 0D & 1D & 2D & 3D \\
\hline \multirow{3}{*}{$\begin{array}{c}\text { Desktop } \\
\text { GIS }\end{array}$} & $\begin{array}{c}\text { ArcGIS } \\
10.8\end{array}$ & & 6 & 16 & 11 & - \\
\hline & QGIS 3.16 & $\begin{array}{c}\text { QGIS } \\
\text { Topology } \\
\text { Rules }\end{array}$ & 6 & 6 & 7 & - \\
\hline & $\begin{array}{l}\text { SuperMap } \\
\text { iDesktop } \\
8 \mathrm{C}\end{array}$ & $\begin{array}{c}\text { SuperMap } \\
\text { Topology } \\
\text { Rules }\end{array}$ & 6 & 14 & 10 & - \\
\hline \multirow{2}{*}{ DBMS } & $\begin{array}{c}\text { PostgreSQL } \\
13 \text { with } \\
\text { PostGIS } 3.2\end{array}$ & $\begin{array}{l}\text { PostGIS } \\
\text { Topology } \\
\text { Rules }\end{array}$ & 0 & 8 & 5 & - \\
\hline & $\begin{array}{l}\text { Oracle with } \\
\text { Oracle } \\
\text { Spatial 21c }\end{array}$ & $\begin{array}{c}\text { Oracle } \\
\text { Topology } \\
\text { Rules }\end{array}$ & \multicolumn{3}{|c|}{$\begin{array}{l}\text { OGC Simple } \\
\text { Features } \\
\text { Specification }\end{array}$} & 8 \\
\hline
\end{tabular}

Table 1. Summary of topology for spatial databases.

The storage of $3 \mathrm{D}$ geometries provides the basis of topological information whereby 3D objects are stored as MultiPatch features in ArcGIS or simple polyhedrons in DBMS such as PostgreSQL with PostGIS and Oracle Spatial. Therefore, no 3D topological primitives are explicitly stored and a limited number of 3D topology rules such as those provided by Oracle Spatial are available on-the-fly. Consequently, the limited 3D topology also limits the execution of 3D spatial queries and query performance (Solihin, Eastman and Lee, 2017).

\section{CUSTOM TOPOLOGICAL EXTENSIONS FOR SPATIAL DATABASES}

Topological information as a spatial property of objects have proven to be crucial in maintaining connectivity information and adjacencies between objects. Its importance can be seen in a variety of spatial applications. Among them such as its use in CityGML (Salleh and Ujang, 2018, 2019), 3D geoinformation (Thomsen et al., 2008), smart city applications (Azri et al., 2018, 2019) and other computing applications (Keling et al., 2017; Löwner, 2013; Mohd et al., 2017). Topological relationships which describe how objects are connected is a simple yet powerful way to preserve topological information. Analyses based on graphs which represent the connectivity of elements also necessitates topological relationships between elements as a prerequisite (den Duijn, Agugiaro, and Zlatanova, 2018).
However, the requirements of topological information depend on the needs of the application and spatial analyses.

Several approaches have been developed as an extension which provides additional topological support for spatial databases. The management of 3D cadastral parcels required accurate representation of topological relationships specifically in determining parcel boundaries. A topological based algorithm was developed as additional topological support for 3D cadastral system to determine complex topological relationships between 3D volumetric parcels or 3DVP (Jaljolie, Riekkinen and Dalyot, 2021). The algorithm utilised directions of vertices to determine the topological relationship of the vertices in relation to other 3D objects (Jaljolie et al., 2021). The algorithm provided further representation for topological relationships between 3D complex objects including concave and hollow objects. Figure 1 illustrates a process of subdividing $3 \mathrm{D}$ parcels at a face using the topological algorithm.

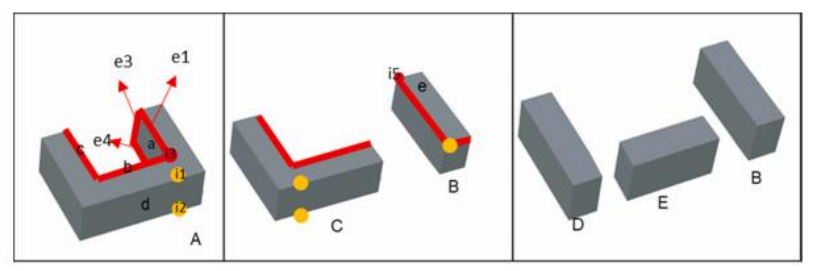

Figure 1. Topological-based approach of subdivision process of 3DVP at a face (Jaljolie et al., 2021).

Another study by Deeken, Wiemann and Hertzberg (2018) integrated a semantic mapping framework (SEMAP) in a PostGIS spatial database which implemented topological relationships to support navigation between $3 \mathrm{D}$ objects in an environment for robotic applications. The additional SEMAP integration includes 3D topological relationships such as containment for 3D objects as well as directional operators which describes objects in relation to other objects in terms of directions (Deeken et al., 2018). In robotic applications, topological relationships and directional operators facilitated exploration of an environment by preserving positional information of objects in relation to each other.

A topology engine (Jaspa) was developed by Llario et al. (2017) in Java for spatial databases to validate complex geographic datasets based on topological rules. Jaspa implemented topological rules that define conditions for relationships between spatial layers to ensure valid geographic data. The topological rules are based on the DE-9IM which determines topological relationships between 2D spatial objects with an additional cluster tolerance parameter (Llario et al., 2017). A similar study by Cuzzocrea and Nucita (2011) developed a Spatial Query Engine for Incomplete Information (I-SQE) which is a spatial reasoning approach to determine topological relationships for spatial databases. The additional topological support was found to facilitate efficient spatial queries from incomplete spatial information (Cuzzocrea and Nucita, 2011).

Additional topology developed in custom extensions varied according to user requirements. For instance, 3D cadastre systems which required 3D topological information implemented a 3DVP topological data model to determine topological relationships (Jaljolie et al., 2021). While some applications only require specific topological information such as containment and intersection, other applications may require more comprehensive topological representation defined by topological rules. Table 3 
summarises the custom extensions developed for spatial databases to provide additional topological support.

\begin{tabular}{|c|c|c|c|c|c|c|c|}
\hline $\begin{array}{l}\text { Custom } \\
\text { Extension }\end{array}$ & Spatial Database & Native Topology & Additional Topology & OD & 1D & 2D & 3D \\
\hline $\begin{array}{l}\text { 3DVP } \\
(\mathbf{2 0 2 1})\end{array}$ & $\begin{array}{l}\text { - 3D Cadastre } \\
\text { (Python-based) }\end{array}$ & - N/A & $\begin{array}{l}\text { - 3D Topological Data Structure } \\
\text { (8 topological relationships) }\end{array}$ & $\sqrt{ }$ & $\sqrt{ }$ & $\sqrt{ }$ & $\sqrt{ }$ \\
\hline $\begin{array}{l}\text { SEMAP } \\
(2018)\end{array}$ & - PostgreSQL & • DE-9IM & $\begin{array}{l}\text { 3D Topological Operators } \\
\text { (Containment \& Intersection) }\end{array}$ & $\sqrt{ }$ & $\sqrt{ }$ & $\sqrt{ }$ & $\sqrt{ }$ \\
\hline $\begin{array}{l}\text { Jaspa } \\
(2017)\end{array}$ & $\begin{array}{l}\text { - PostgreSQL } \\
\text { - Oracle }\end{array}$ & $\begin{array}{l}\text { - PostGIS } \\
\text { Topology } \\
\text { Rules } \\
\end{array}$ & $\begin{array}{l}\text { - Topological Rules } \\
\text { (67 rules) }\end{array}$ & $\sqrt{ }$ & $\sqrt{ }$ & $\sqrt{ }$ & $\sqrt{ }$ \\
\hline $\begin{array}{l}\text { I-SQE } \\
(2011)\end{array}$ & - MySQL & - N/A & $\begin{array}{l}\text { - Topological Composition } \\
\text { Function ( } 8 \text { topological } \\
\text { relationships) }\end{array}$ & $\sqrt{ }$ & $\sqrt{ }$ & $\sqrt{ }$ & $x$ \\
\hline
\end{tabular}

Table 3. Custom topological extensions for spatial database.

\section{DISCUSSION ON 3D TOPOLOGICAL SUPPORT IN SPATIAL DATABASES}

In terms of data storage, complex objects stored in spatial databases are composed of geometries such as $0 \mathrm{D}$ points, 1D lines, 2D surfaces and 3D polyhedrons. However, topological support in spatial databases have been limited to only $2 \mathrm{D}$ topology or few topology rules for 3D objects. Therefore, it is sufficient for $2 \mathrm{D}$ topology rules to be used in spatial databases. However, the interpretation of topological relationships is limited to $2 \mathrm{D}$ and does not describe $3 \mathrm{D}$ topological interactions. This is supported by Ellul (2006) who found that analysis results will remain in $2 \mathrm{D}$ if the topology is in $2 \mathrm{D}$. $3 \mathrm{D}$ objects must also be decomposed into lower dimension objects in order to adhere to the $2 \mathrm{D}$ topology rules. This is counter-productive to the capabilities of the spatial database in handling 3D objects.

The previous section has put forth examples of topological extensions developed to provide additional topological support for spatial databases. The extensions were developed according to specific requirements of the application. The approaches to implement topology in a spatial database included the use of 3D topological data structure, topological operators or functions and topological rules. The implementation of topology in spatial database is also determinant on a number of factors such as analysis requirements, storage space, query efficiency and others. Topological support in the simplest form is the topological model or schema which is implemented by most commercial and opensource spatial database. Similar to geometric specifications provided by Open Geospatial Consortium Simple Feature Access (OGC-SFA), a schema for topology can also be utilised by spatial database to represent the topological properties of objects. However, the implementation of the model can vary from a limited representation of "relates" between two objects up to a more detailed but hierarchical representation of topological relationships. Spatial operators that execute specific functions such as determining intersections or containment can also be implemented in maintaining topology.

In attempting to maintain topological properties of objects as accurately as possible, a topological data structure can be implemented by spatial database to maintain topological properties. A topological data structure explicitly stores topological primitives and relationships (Ohori, 2015). However, due to its extensive and complex nature, topological data structures are often developed separate to the spatial database as it requires a lot of space. Nonetheless, the use of a topological data structure provides a comprehensive foundation for spatial analysis and can be extended up to higher dimensions of data.
Currently, the middle ground of topological support in spatial database is the use of topological rules that define valid topological relations. The implementation of topological rules often covers basic relations to achieve the requirements of the spatial database and have limited expansion capabilities. However, more complex analysis will require implementing more comprehensive topological rules. Even so, comprehensive or detailed topological rules will not be as complex as implementing a topological data structure due to the focus only being on the topological relationships and not on all of the topological primitives or properties.

\section{CONCLUSION}

This paper provided an overview on 3D topological support in spatial databases. Current topological support for commercial and open-source spatial databases implemented topological rules that can support 2D topology for 2D objects. In addition, 2D topological rules could also maintain topological information for $3 \mathrm{D}$ objects by decomposing the $3 \mathrm{D}$ objects into $2 \mathrm{D}$ or lower dimension objects. The topological rules implemented in spatial databases also differ in comprehensiveness where not all valid topological interactions between spatial objects are defined. The representation of valid topological relationships between spatial objects of different dimensions are also limited. However, more complex analysis that requires connectivity, containment and adjacency information requires 3D topological representation of spatial objects.

Additional implementations of topological models, data structures, operators and rules were developed as an effort to improve topological support for spatial databases. Applications that required specific topological information utilised topological operators or topological models as a lightweight approach in maintaining topological information tailored to the users' requirements. On the other hand, 3D topological data structures allowed a comprehensive preservation of topological primitives from which 3D topological information and relationships can be extracted for 3D spatial analysis. Apart from that, 3D topological rules were also implemented to define valid topological interactions between 3D spatial objects without decomposing the objects into geometries of lower dimensions. This ensured valid topological interactions between 3D spatial objects in ensuring accurate spatial analysis without the need to explicitly store all the topological primitives of objects.

Parallel to how different 3D applications may require different levels of comprehensive topological information, the approaches implemented to improve topological support in spatial databases 
also varies according to its topological requirements. 3D applications that require detailed topological information may also require voluminous storage and higher computational efficiency to facilitate complex 3D topological data structures. As storage is a concern for spatial databases, 3D topological rules can be implemented as a compromise that does not require much storage while still maintaining comprehensive $3 \mathrm{D}$ topological information. Thus, the appropriate 3D topological support should be provided in spatial databases to meet 3D analysis requirements and obtain accurate results.

\section{ACKNOWLEGEMENTS}

This research was supported by Ministry of Education (MOE) through Fundamental Research Grant Scheme (FRGS/1/2021/WAB07/UTM/02/2). Partial funding was also provided by UTM Research University Grant Vot Q.J130000.3652.02M57.

\section{REFERENCES}

Atazadeh, B., Kalantari, M., Rajabifard, A., Ho, S., Champion, T., 2017. Extending a BIM-based data model to support 3D digital management of complex ownership spaces. International Journal of Geographical Information Science, 31, 499-522.

Azri, S., Ujang, U., Abdul Rahman, A., 2019. 3D Geo-clustering for Wireless Sensor Network in Smart City. Int. Arch. Photogramm. Remote Sens. Spatial Inf. Sci., XLII-4/W12, 11-16.

Azri, S., Ujang, U., Abdul Rahman, A., 2018. Dendrogram Clustering for 3D Data Analytics in Smart City. Int. Arch. Photogramm. Remote Sens. Spatial Inf. Sci., XLII-4/W9, 247253.

Biljecki, F., Ledoux, H., Stoter, J., 2017. Does a Finer Level of Detail of a $3 D$ City Model Bring an Improvement for Estimating Shadows?, In Abdul-Rahman, A. (Ed.), Advances in 3D Geoinformation. Springer International Publishing, Cham, 3147.

Cuzzocrea, A., Nucita, A., 2011. Enhancing Accuracy and Expressive Power of Range Query Answers Over Incomplete Spatial Databases via a Novel Reasoning Approach. Data \& Knowledge Engineering, 70(8), 702-716.

Deeken, H., Wiemann, T., Hertzberg, J., 2018: Grounding Semantic Maps in Spatial Databases. Robotics and Autonomous Systems, 105, 146-165.

Den Duijn, X., Agugiaro, G., Zlatanova, S., 2018. Modelling Below- And Above-Ground Utility Network Features with the CityGML Utility Network ADE: Experiences from Rotterdam. ISPRS Annals of Photogrammetry, Remote Sensing and Spatial Information Sciences, 4(4/W7).

Döllner, J., Hagedorn, B., 2007. Integration of GIS, CAD and BIM data with service-based virtual $3 D$ city models. In Integrating Urban GIS, CAD and BIM Data By Service-Based Virtual 3D City Models, 28-37.

Ellul, C., Haklay, M., 2006. Requirements for Topology in 3D GIS. Transactions in GIS, 10(2), 157-175.

ESRI, 2010. ArcGIS Geodatabase Topology Rules - Topology Rules | Resources ArcGIS. https://desktop.arcgis.com/en/arcmap/latest/managedata/topologies/topology-basics.htm.

Howari, F. M., Ghrefat, H., 2021. Chapter 4 - Geographic information system: spatial data structures, models, and case studies. In A.-M. O. Mohamed, E. K. Paleologos, \& F. M. Howari (Eds.), Pollution Assessment for Sustainable Practices in Applied Sciences and Engineering, 165-198.

Jaljolie, R., Riekkinen, K., Dalyot, S., 2021. A Topologicalbased Approach for Determining Spatial Relationships of Complex Volumetric Parcels in Land Administration Systems. Land Use Policy, 109, 105637.

Jovanović, D., Milovanov, S., Ruskovski, I., Govedarica, M., Sladić, D., Radulović, A., Pajić, V., 2020. Building Virtual 3D City Model for Smart Cities Applications: A Case Study on Campus Area of the University of Novi Sad. ISPRS International Journal of Geo-Information, 9.

Keling, N., Mohamad Yusoff, I., Lateh, H., Ujang, U., 2017. Highly Efficient Computer Oriented Octree Data Structure and Neighbours Search in 3D GIS. In Abdul-Rahman, A. (Ed.), Advances in 3D Geoinformation. Springer International Publishing, Cham, 285-303.

Knoth, L., Atazadeh, B., Rajabifard, A., 2020. Developing a new framework based on solid models for 3D cadastres. Land Use Policy, 92, 104480.

Ledoux, H., 2008. The Kinetic 3D Voronoi Diagram: A Tool for Simulating Environmental Processes, In Oosterom, P., Zlatanova, S., Penninga, F., Fendel, E.M. (Eds.), Advances in 3D Geoinformation Systems. Springer Berlin Heidelberg, 361-380.

Löwner, M.-O., 2013. On Problems and Benefits of 3D Topology on Under-Specified Geometries. In Geomorphology, Progress and New Trends in 3D Geoinformation Sciences. Springer Berlin Heidelberg, 155-170.

Maguire, D. J., 2008. ArcGIS: General Purpose GIS Software System. In S. Shekhar \& H. Xiong (Eds.), Encyclopedia of GIS, 25-31. Boston, MA: Springer US.

Martinez-Llario, J., Coll, E., Núñez-Andrés, M., FemeniaRibera, C., 2017. Rule-based Topology System for Spatial Databases to Validate Complex Geographic Datasets. Computers \& Geosciences, 103, 122-132.

McDonnell, R., Kemp, K., 1995. International GIS dictionary. John Wiley \& Sons.

Mella, E., Rodríguez, M. A., Bravo, L., Gatica, D., 2019. Query rewriting for semantic query optimization in spatial databases. GeoInformatica, 23(1), 79-104.

Mohd, Z.H., Ujang, U., Choon, T.L., 2017. Heritage House Maintenance using 3D City Model Application Domain Extension Approach. International Archives of the Photogrammetry, Remote Sensing \& Spatial Information Sciences, 42.

Ohori, K. A., Ledoux, H., Stoter, J., 2015. An Evaluation and Classification of $\mathrm{nD}$ Topological Data Structures for the Representation of Objects in a Higher-dimensional GIS. International Journal of Geographical Information Science, 29(5), 825-849. 
QGIS, 2021. 25.2.5. Topology Checker Plugin - 25.2. Using QGIS Core Plugins - 25. Plugins - QGIS User Guide | QGIS Desktop User Guide/Manual (QGIS 3.16).

https://docs.qgis.org/3.16/en/docs/user_manual/plugins/core_pl ugins/plugins_topology_checker.html.

Salleh, S., Ujang, U., Azri, S., 2021. Virtual 3D Campus for Universiti Teknologi Malaysia (UTM). ISPRS International Journal of Geo-Information, 10(6), 356.

Salleh, S., Ujang, U., 2019. Characteristics of a 3D Topological Data Structure for Buildings in CITYGML. International Graduate Conference of Built Environment and Surveying (GBES 2019). UTM Skudai, Malaysia, 1-6.

Salleh, S., Ujang, U., 2018. Topological information extraction from buildings in CityGML. IOP Conference Series: Earth and Environmental Science, 169, 012088.

Solihin, W., Eastman, C., Lee, Y.-C., 2017. Multiple representation approach to achieve high-performance spatial queries of 3D BIM data using a relational database. Automation in Construction, 81, 369-388.

SuperMap, 2017. Topology Rules - Topology - Data Processing - Features - WebHelp | iDesktop9D.

https://enonline.supermap.com/iDesktop9D/WebHelp/Features/ DataProcessing/Topology/TopoRule.htm\#222.

Thomsen, A., Breunig, M., Butwilowski, E., Broscheit, B., 2008. Modelling and managing topology in $3 D$ geoinformation systems. In Advances in 3D Geoinformation Systems. Springer, 229-246.

Ujang, U., Azri, S., Zahir, M., Abdul Rahman, A., Choon, T.L., 2018. Urban Heat Island Micro-Mapping via 3D City Model. Int. Arch. Photogramm. Remote Sens. Spatial Inf. Sci., XLII-4/W10, 201-207.

Wan Abdul Basir, W.N.F., Majid, Z., Ujang, U., Chong, A., 2018. Integration of GIS and BIM Techniques in Construction Project Management - A Review. Int. Arch. Photogramm. Remote Sens. Spatial Inf. Sci., XLII-4/W9, 307-316.

Winstanley, A. C., Mooney, P., 2020. Spatial Databases. In A. Kobayashi (Ed.), International Encyclopedia of Human Geography (Second Edition), 383-386.

Worboys, M. F., Duckham, M., 2004. GIS: A Computing Perspective. CRC press.

Yue, P., Tan, Z., 2018. GIS Databases and NoSQL Databases. In B. Huang (Ed.), Comprehensive Geographic Information Systems, 50-79. 